\title{
Apoyo familiar y desempeño académico de tres contextos educativos en Sonora, nivel primaria
}

Eneida Ochoa Ávila, ${ }^{1}$ Rafael Octavio Félix Verduzco, ${ }^{1}$ Alejandrina Limón Fernández y Mónica Cecilia Dávila Navarro ${ }^{1}$

\section{Introducción}

Akmal y Larsen (2004) señalan que las escuelas que involucran a los padres en programas orientados al mejoramiento académico de sus hijos aumentan la probabilidad de que los estudiantes tengan un buen desarrollo académico en el cambio de un grado escolar a otro. En relación con esto, la Organización de las Naciones Unidas para la Educación, la Ciencia y la Cultura (UNESCO, 2004) aboga por la relación familia-escuela, justificando esta necesidad en tres puntos importantes: a) El reconocimiento de que los padres son los primeros educadores de sus hijos e hijas; b) el impacto positivo que puede tener una educación temprana de calidad en el desarrollo y aprendizaje de los niños; y c) la familia como un espacio privilegiado para lograr una ampliación de la cobertura de la educación de la primera infancia.

La evidencia empírica actual reconoce la importancia del contexto social y las personas que lo componen y su relación

1 Instituto Tecnológico de Sonora. 
con el desempeño académico del alumno(a) (Ceballos, 2006). Al respecto, diversos estudios hablan sobre la relación de los factores escolares y los aspectos emocionales del niño (personales, sociales y familiares) como responsables de su logro académico, señalando que sus resultados son producto no sólo de las competencias del alumno sino también de la interacción de los recursos aportados por la escuela y el hogar (Ruiz, 2001; Sánchez, 2006). Asimismo, también se menciona que las variables familiares son las principales predictoras del desempeño académico (Brunner y Elacqua, 2003).

Así pues, parece evidente la importancia que tiene para el aprendizaje el que las relaciones entre los padres de alumnos y los profesionales educativos sean fluidas (Alomar, 2006; Rolla, Arias, Villers y Show, 2005). Por tal motivo, el objetivo del presente trabajo es describir el apoyo familiar percibido por los padres y los alumnos sobre las materias de matemáticas y español, así como el nivel de desempeño académico presentado por los alumnos de sexto grado en tres contextos educativos.

\section{Método}

\section{Participantes}

La población participante estuvo conformada por 610 personas de ambos sexos, 305 alumnos de educación básica divididos en diferentes contextos educativos, 153 en privado, 67 en urbano y 85 en rural. Asimismo, se trabajó con los 305 padres y tutores de dichos estudiantes.

\section{Instrumentos}

Se utilizó la Escala de Percepción de Apoyo Familiar en dos versiones de Domínguez (2009) con opciones de respuesta de 0 a 4 y con indicadores de consistencia interna por contexto por arriba de .86. Los cuestionarios para alumnos y padres incluyen los mismos indicadores y criterios de valoración. Cada escala 
consta de 19 preguntas divididas en cuatro subescalas: a) Asistencia o apoyo en tareas escolares, b) tiempo y espacio proporcionado para el estudio, c) comunicación regular con las figuras docentes, y d) repaso y evaluación.

Para evaluar el desempeño académico en Español se utilizó el instrumento de evaluación de dos competencias de Español de sexto grado de primaria (comprensión lectora y producción de textos), construido y validado por Bazán, Barrera y Vega (2013), de acuerdo con la estructura curricular propuesta en la reforma integral de la educación básica para el sexto grado de primaria en Español (SEP, 2009), el cual cuenta con indicadores de consistencia interna por contexto por arriba de .85 (Ferrari, 2014). Este instrumento de evaluación incluye 36 ejercicios o actividades de evaluación, agrupados en 16 series divididas en dos grandes competencias. Cada ítem cuenta con 5 valores posibles de calificación de 0 a 4.

Para Matemáticas, se utilizó el instrumento de evaluación de competencias matemáticas, construido y validado por Bazán, Hernández, Ocampo y Vega (2011), de acuerdo con la estructura curricular propuesta en la reforma integral de la educación básica para el sexto grado de primaria en Español (SEP, 2009), el cual cuenta con indicadores de consistencia interna por contexto por arriba de .85 (Ferrari, 2014). El instrumento se encuentra conformado por 12 ejercicios que evalúan cuatro competencias matemáticas: a) "Comunicar información matemática" (cIM), "Validar procedimientos y resultados" (VPR), "Resolver problemas de manera autónoma" (RPMA), y "Manejar técnicas eficientemente" (MTE). Cada ítem contaba con cinco valores posibles de calificación (0-4).

\section{Procedimiento}

Se tomó la información necesaria para realizar los análisis estadísticos de una base de datos alimentada de diferentes instrumentos aplicados para la realización de un estudio más amplio, del cual se desprende el presente documento. Una vez seleccionada la información para trabajar de la base de datos en SPSS, 
se procedió a realizar los análisis de frecuencias de cada uno de los instrumentos y, finalmente, se redactaron los resultados y discusión, los cuales se muestran a continuación.

\section{Resultados}

Los resultados arrojados de los análisis estadísticos para el presente documento presentan, en primera instancia, un análisis de frecuencias del instrumento de desempeño en español, en el cual se puede apreciar cómo el desempeño de los tres contextos para la competencia de comprensión lectora se mantiene cargado hacia el rango de 0 en una escala de 0 a 4; sin embargo, en el aprendizaje esperado de corregir errores ortográficos se muestra un marcado dominio del aprendizaje en la parte privada con puntuación de 4, mientras que la población urbana presenta una división homóloga entre quienes dominan y quienes no dominan dicho aprendizaje con puntuaciones de 0 y 4; sin embargo, como ya se mencionó, la población de los tres contextos presenta un bajo desempeño para esta competencia.

Para la competencia de producción de textos, se aprecia cómo en el contexto rural el dominio de la misma es completamente bajo (puntaje de 0); sin embargo, en el contexto urbano y privado se presenta una mejora en el comportamiento de la población para los aprendizajes de redactar preguntas abiertas y cerradas con puntuaciones de 3 y 4 , así como para elaborar carta con puntuaciones de 2 a 4; sin embargo, los tres contextos presentan un bajo desempeño para esta competencia.

Sobre la prueba de matemáticas se aprecia una mayor dispersión en los puntajes de los tres contextos, donde aun así el contexto rural presenta un muy bajo desempeño con puntuaciones por aprendizaje esperado entre 0 y 1 en su mayoría; sólo los aprendizajes esperados de elegir representaciones gráficas lograron puntuaciones de 3 y 4 para este contexto; por otro lado, el desempeño mejora en el contexto urbano y privado, alcanzando puntuaciones de 2 a 4 por aprendizaje esperado; sin embargo, la competencia con puntuación más alta (Resolver problemas de 
manera autónoma) se obtuvo en el contexto privado, con calificación de 4.

Pasando a la percepción de apoyo familiar de los niños en español, puede apreciarse cómo los alumnos de educación rural y urbana perciben más el apoyo de sus padres, presentando calificaciones para las cuatro categorías entre 3 y 4 en una escala del 0 al 4, mientras que los participantes de la privada sólo perciben el apoyo de sus padres en las categorías 2 y 3.

Siguiendo con la percepción de los niños, pero en este caso para Matemáticas, se puede apreciar los mismos resultados que en Español en su mayoría; sin embargo, dentro del área rural, la categoría 2, correspondiente a "Proporcionar el tiempo y espacio para el estudio", muestra cómo solamente la mitad de los niños perciben un apoyo, obteniendo puntuaciones de 3 a 4.

En lo referente a los padres, se presenta un comportamiento similar a la percepción de los niños; sin embargo, en el contexto rural, la categoría "Evaluación y repaso" presenta calificaciones bajas para los padres; sin embargo, fuera de este aspecto la dinámica coincide con la percepción de los niños sobre el apoyo en Español.

Finalmente, el apoyo percibido por parte de los padres para Matemáticas muestra cómo algunos padres no presentan un apoyo en la categoría "Evaluación y repaso", y para la categoría "Proporcionar el tiempo y espacio para el estudio", el apoyo está dividido dentro del área rural, con puntuaciones entre 0 y 1 , así como 3 y 4 , respectivamente.

\section{Discusión}

Los resultados presentados muestran una exploración inicial de los tres contextos sobre la distribución de las frecuencias de las personas participantes de este estudio en los instrumentos aplicados para medir desempeño académico y percepción de apoyo familiar en alumnos y padres. Dichos resultados muestran una coincidencia de bajos resultados en las pruebas de desempeño de manera general, aunque en lo particular algunos de los 
aprendizajes muestran calificaciones medias y altas de forma particular, lo cual es un aspecto por considerar para análisis más profundos sobre las variables en estudio con esta población.

Por otro lado, en lo referente a percepción de apoyo familiar, los resultados muestran, en lo que respecta a la percepción de los niños, un mayor apoyo por parte de los padres para los contextos urbano y rural que en los de contexto privado, donde el apoyo se registró en la comunicación con los maestros y en proporcionar espacios y materiales tanto en Español como en Matemáticas. Para la percepción de los padres, los resultados coinciden en su mayoría con los de los alumnos; sin embargo, en el contexto rural existe una división entre los padres que están en contacto con los docentes y los que no lo están, y se presentó poco apoyo en evaluación y repaso tanto en Matemáticas como en Español.

Lo anterior nos brinda un panorama para realizar próximos análisis sobre la relación de los bajos desempeños sobre la percepción de apoyo de alumnos y padres en Matemáticas y Español, con lo cual se observa que, en general, tanto los padres como las madres, participan poco en las actividades educativas de sus hijos.

\section{Referencias}

Akmal, T. y Larsen, D. (2004). Keeping History from Repeating Itself: Involving Parents about Retention Decisions to Support Student Achievement. Research in Middle Level Education, 27(2), 1-14.

Alomar, B. (2006). Personal and Family Paths to Pupil Achievement. Social Behavior and Personality, 34(8), 907-922. Recuperado de http://www.sbp-journal.com/index.php/sbp/ login?source=\%2Findex.php\%2Fsbp\%2Farticle\%2Fv.

Bazán, A., Barrera, D. y Vega, N. (2013). Validación de constructos de competencias de lectura y producción de textos en los inicios de la generalización de la Reforma en la primaria Mexicana. REICE. Revista Iberoamericana sobre 
Calidad, Eficacia y Cambio en Educación, 11(4), 61-76. Recuperado de http://www.rinace.net/reice/numeros/arts/ vol11num4/art4.pdf.

Bazán, A., Hernández, F., Ocampo, C. y Vega, N. (2011). Diseño y validación de una evaluación de competencias matemáticas para sexto grado de primaria. Trabajo presentado en el XIX Congreso Mexicano de Psicología. Cancún, México.

Brunner, J. y Elacqua, G. (2003). Informe capital humano en Chile. Santiago: La Araucana.

Ceballos, E. (2006). Dimensiones de análisis del diagnóstico en educación: El diagnóstico del contexto familiar. Revista Electrónica de Investigación y Evaluación Educativa, 12(1). Recuperado de http://www.uv.es/RELIEVE/v12n1/ RELIEVE12n1_4.htm.

Domínguez, L. (2009). Apoyo familiar y desempeño en lengua escrita en escolares de quinto grado de primaria (Tesis de Licenciatura en Psicología, inédita). Universidad Autónoma del Estado de Morelos, Cuernavaca, Morelos.

Ferrari, A. (2014). Validación de instrumentos de evaluación por competencias en Matemáticas y Español en sexto grado de primaria en Morelos y Sonora (Tesis de Licenciatura en Psicología, inédita). Universidad Autónoma del Estado de Morelos, Cuernavaca, Morelos.

Rolla, A., Arias, M., Villers, R. y Show, C. (2005). The Importance of Reading Difficulties and Family in Teachers Decisions to Retain Children: A Case Study in Costa Rica. Aula Abierta, 85, 147-164.

Ruiz, C. (2001). Factores familiares vinculados al bajo rendimiento. Revista Complutense de Educación, 12(1), 81-113. Recuperado de http://revistas.ucm.es/index.php/RCED/article/viewFile/RCED0101120081A/16850.

Sánchez, P. (2006). Discapacidad, familia y logro escolar. Revista de Educación, 40(2), 1-10. Recuperado de http://www. rieoei.org/1538.htm.

Secretaría de Educación Pública. (2009). Programas de Estudio 2009 Sexto Grado Educación Básica Primaria. México: SEP. 
Organización de las Naciones Unidas para la Educación, la Ciencia y la Cultura. (2004). Participación familiar en la educación infantil latinoamericana. Santiago de Chile: Oficina Regional para la Educación de América Latina y el Caribe-UNESCO. 